\title{
MIXING OF IMMISCIBLE FLUIDS IN CHAOTIC FLOWS AND RELATED ISSUES
}

\author{
Progress Report \\ for Period June 1, 1992 - May 31, 1993 \\ Julio M. Ottino \\ Northwestern University \\ Evanston, Illinois 60208-3120
}

March 1993

Prepared for

THE U. S. DEPARTMENT OF ENERGY AGREEMENT NO. DE-FG02-91ER14182 
This report was prepared as an account of work sponsored by the United States Government. Neither the United States nor the Department of Energy, nor any of their employees, nor any of their contractors, subcontractors, or their employees, makes any warranty, express or implied, or assumes any legal liability or responsibility for the accuracy, completeness, or usefulness of any information, apparatus, product or process disclosed or represents that its use would not infringe privately-owned rights. 


\section{MIXING OF IMMISCIBLE FLUIDS AND RELATED IsSUES}

J.M. Ottino

Northwestern University, Evanston

March 30, 1993

\section{ORIGINAL OBJECTIVES AND SETTING}

Before presenting the results obtained during the last year and the plans for the final year of the grant, 1993-94, let us review the the original objectives of the proposal The basic goal of this work was to obtain "basic understanding of mixing of immiscible fluids" leading to "the determination of flow conditions which result in efficient breakup and dispersion of one mass of fluid in the bulk of another". Related issues were "the prediction of the morphological structures and drop size distribution for a given set of operating conditions". The primary motivation for these investigations was to produce basic knowledge leading to increased understanding of "industrial processes involving blending, agitation, emulsification, and dissolution."

\section{REPORT: RESULTS OBTAINED}

Work was carried out in several inter-related areas:

(i) stretching and breakup of filaments under super-critical conditions in chaotic flows,

(ii) analysis of details of breakup and sub-satellite formation at small scales,

(iii) understanding of the statistics of drop size distributions produced for different viscosity ratios.

Issues (i-ii) form the basis for the understanding of (iii). Experimental studies were carried out in several classes of carefully controlled flows using well-characterized fluid systems spanning a wide range of viscosities and surface tensions. The bulk of the experiments were conducted using viscous Newtonian fluids but a number of experiments using viscoelastic fluids were conducted as well. Even though we had initially restricted our objectives to computational studies of Newtonian fluids, we nevertheless conducted extensive investigations involving viscoelastic fluids. This constitutes a new area of investigation -- denoted (iv) -- under this proposal.. 
Work under area (i) has been virtually completed, and al though several issues await clarification, no further work is planned in this area. 1 Work under (ii) has been completed as well. A summary of our results in area (ii) based on of applications of boundary integral techniques is given in:

M. Tjahjadi, H.A. Stone, and J.M. Ottino, Satellite and Sub-Satellite Formation in Capillary Breakup, J. Fluid Mech., 243, 297-317 (1992).

Results under (iii) show that the drop size distribution is consistent with scaling, assuming that moderately extended filaments behave passively in chaotic flows. This work builds on our previous studies reported in F.J. Muzzio, M. Tjahjadi, and J.M. Ottino, Self-Similar Drop Size Distributions Produced by Breakup in Chaotic Flows, Phys. Rev. Lett., 67, 54 57, 1991.

A presentation of our results in this area appears in: F.J. Muzzio, P.D. Swanson, and J.M. Ottino, Mixing Distributions Produced by Multiplicative Stretching in Chaotic Flows, Int. J. Chaos and Bifurcations, 2, 37-50 (1992).

Related results, but extending the investigations into quantification of mixing appear in:

F.J. Muzzio, C. Meneveau, P.D. Swanson, and J.M. Ottino, Scaling and multifractal properties of mixing in chaotic nows, Phys. Fluids A, 4, 1439-1456 (1992).

A presentation of our results involving mixing of viscoleastic fluids - area (iv) - has been prepared for publication:

T.C. Niederkorn and J.M. Ottino, Mixing of viscoelastic fluids in time-periodic flows, $J$. Fluid Mech.. submitted for publication.

${ }^{1}$ Most of the important results in this area appear in M. Tjahjadi and J.M. Ottino, Stretching and breakup of droplets in chaotic flows, J. Fluid Mech., 232, 191-219, 1991. 
An overall summary of most of our results through mid 1992 - excluding viscoelastic fluids - is given in:

J. M. Ottino, F. J. Muzzio, M. Tjahjadi, J. G. Franjione, S. C. Jana, H. A. Kusch, Chaos, symmetry, and self-similarity: exploiting order and disorder in mixing processes, Science, 257, 754-760 (1992).

A summary of our work in mixing and its connection with chaos, written from a distinctively engineering viewpoint, appears in:

J.M. Ottino, New Applications of Chaos in Chemical Engineering: Intuition versus Prediction, in Applied Chaos, Edited by J.H. Kim and J. Stringer, Chapter 5, pp. 143 174, New York: J. Wiley, 1992.

\section{PLANS (1993 ONWARDS)}

We plan to extended our investigations in two new directions: (v) Mixing of equal volumes of fluids using either the marker and cell technique or the boundary integral element method (BIEM); and (vi) Investigation of the role of rheology, especially shear thinning effects, in chaotic mixing processes [ $(v)$ focuses on the early stages of the mixing process, before scenario breakup takes place.] Studies under item (v) complement those conducted under (iv) and comprise both experimental and computational investigations of steady and time-periodic flow of a constant viscosity elastic fluid in a two-dimensional eccentric cylindrical geometry. The work on quantification of mixing - multifractals - will be phased out. However, another area -- an of fspring of (ii) -- will be pursued further. This work involves satellite formation computations for the calculation of interfacial tension between two immiscible viscous fluids. A paper is in this area is ready for submission:

M. Tjahjadi, A.A. Stone, and J.M. Ottino, Estimation of interfacial tension from breakup and relaxation of fluid filaments, to be submitted to the AIChE Journal.

A short description of the fundamentals of the work to be carried out in area (v) follows. This work involves the description of mixing of equal volumes of fluids in a cavity. The problem is essentially one of following the evolution of the interface between the two fluids. The work will be initially restricted to steady flows but the final objective is to extend the results to chaotic flows. For this problem we plan to use either the marker and 
cell technique and/or boundary integral techniques. We already have experience using BIEM and our initial efforts will probably be directed along these lines. The equations to solve are:

$$
\mu_{i} \nabla^{2} u_{i}=\nabla P_{i}, \quad \nabla \cdot u_{i}=0 \quad(i=1,2) .
$$

The viscosity ratio is denoted as $p=\mu_{1} / \mu_{2}$ and the interfacial tension between the two fluids is $\sigma$. Points in both fluid domains are denoted by the position vector $\mathbf{x}$ and points at the fluid-fluid interface are denoted by $x_{s}$. Let $T_{1}=-p_{1} I+2 \mu_{1} D_{2}$ and $T_{2}=-p_{2} I+2 \mu_{2} D_{2}$, where $D=(1 / 2)\left[\nabla v+(\nabla v)^{T}\right]$, denote the stress tensors in the phases 1 and 2 and $\nabla_{s} \cdot n$ the mean curvature of the fluid-fluid interface with the local normal $\mathbf{n}$ directed from 1 to 2 . The boundary condition at the interface is

$$
n \cdot\left(T_{2}-T_{1}\right)=\text { on }\left(\nabla_{s} \cdot n\right) \quad \text { for } x=x_{s}
$$

along with the kinematic condition

$$
d \mathbf{x}_{s} / d \mathbf{t}=\mathbf{n}(\mathbf{u} \cdot \mathbf{n})
$$

Pressure terms are of the same order of magnitude as viscous terms. Moreover, if the length scales associated with velocity changes are normalized by $\delta_{v}$, length scales associated with curvature changes normalized by $\delta_{s}$ (a typical striation thickness), the stress condition reduces to:

$$
\mathbf{n} \cdot\left(\mathbf{T}_{2}^{\prime}-p \mathbf{T}_{1}^{\prime}\right)=(1 / C a) \mathbf{n}\left(\nabla_{s}^{\prime} \cdot \mathbf{n}\right)
$$

where $C a=\left[\mu_{2} \mathrm{~V} \delta_{s}(t)\right] /\left(\delta_{v}{ }^{2} \sigma\right)$ is the capillary number and the primed quantities denote dimensionless variables. In chaotic flows $\delta_{s}(t)=\delta_{s}(0) \exp (-\kappa t)$, where $\kappa$ is a Liapunor exponent. It is therefore apparent that the importance of the viscous forces increases as a mixing proceeds. Under most conditions of interest the capillary number is initially effectively infinity and interfacial tension plays no role. We will start our investigations by: focussing on this case first. 
There are few studies in this area. In fact, we know of only one clear-cut attempt to examine precisely this issue. In 1974 Bigg \& Middleman 3 considered a square cavity filled with equal volumes of fluids having a viscosity ratio $p$. A constant speed top plate drove the 2-d flow. The bulk of the investigation was computational, using the so-called "marker and cell" technique, and Bigg \& Middleman were able to show that the interface rapidly deforms in a complex way. The computed interface geometry was verified with an experiment. Their studies were necessarily low-resolution and limited to small deformation times. Even though the explored parameter space was small, the computations suggested that the viscosity ratio $p$ plays an important role in the deformation of the interface.

The fluid interface becomes highly convoluted quickly, so in order to simulate the interface for even moderate deformation times, high computational resolution is needed. With a finite difference method, it is ultimately impossible to track the interface as it folds. In fact, in chaotic flows it is nearly impossible to con. nute accurately length stretches of material lines for length stretches greater than $10^{4}$. Another possibility - considerably more speculative - involves the use of cellular automata techniques.

BOUNDARY INTEGRAL METHOD: The boundary integral method ${ }^{4}$ is especially suited to problems involving free boundaries. Briefly, the velocity at any point in fluids 1 and 2 is given by the integral identities

$$
\begin{aligned}
& \mathrm{u}_{\mathrm{e}}(\mathrm{x})=-\int_{\mathrm{S}} \mathrm{n} \cdot \mathbf{T}_{\mathrm{e}} \cdot \mathbf{J} d S(\mathbf{y})+\int_{\zeta} \mathrm{n} \cdot \mathrm{K} \cdot \mathbf{u}_{\mathrm{e}} d S(\ddot{j}) \\
& \left.\mathrm{u}_{\mathrm{i}}(\mathrm{x})=\int_{\mathrm{n}} \mathrm{n} \cdot \mathrm{T}_{\mathrm{i}} \cdot \mathbf{J} d S(\mathrm{y})-\int_{\mathrm{n}} \mathrm{K} \cdot \mathrm{K} \cdot \mathrm{u}_{\mathrm{i}} d S(\mathrm{y})\right)
\end{aligned}
$$

where

\footnotetext{
${ }^{3}$ Bigg, D. and Middleman, S. . Laminar mixing of a pair of fluids in a rectangular cavity: Ind. Eng. Chem. Fundam., 13, 184-190, 1974.

4Pozrikidis, C. Boundary Integral and Singularity Methods for Linearized Viscous Flow, Cambridge University Press, 1992.
} 


$$
\begin{aligned}
& J=\frac{1}{8 \pi}\left[\frac{1}{|x-y|}+\frac{(x-y)(x-y)}{|x-y|^{3}}\right] . \\
& K=-\frac{3}{4 \pi} \frac{(x-y)(x-y)(x-y)}{|x-y|^{5}} .
\end{aligned}
$$

In these equations, $S$ represents the interface between the fluids and $\mathbf{y}$ is the integration variable. Taking the limit $\mathbf{x} \rightarrow \mathbf{x}_{s}$ and making use of the interfacial conditions, the above equations generate an integral equation of the second kind for the interfacial velocity $\mathbf{u}_{\mathbf{s}}\left(\mathbf{x}_{\mathbf{s}}\right)$ :

$$
\frac{1}{2}(1+\mathrm{p}) \mathbf{u}_{\mathrm{s}}\left(\mathbf{x}_{\mathrm{s}}\right)+(1-\mathrm{p}) \int \mathrm{n} \cdot \mathbf{K} \cdot \mathbf{u}_{\mathrm{s}} d S(\mathbf{y})=-\int \mathbf{n} \cdot \mathbf{J}\left(\boldsymbol{\nabla}_{\mathbf{s}} \cdot \mathbf{n}\right) d S(\mathbf{y})
$$

We have recently used this method to compute the evolution and multiple breakup of a fluid filament. The first results in this area will be available be the end of the summer.

OTHER ACTIVITIES DIRECTLY RELATED TO THIS GRANT:

A mixing and chaos course, "Modern Analytical Techniques for Viscous Mixing and Dispersion", directed primarily to industry, has been prepared and will be given May 2-4 in Evanston, Illinois. 

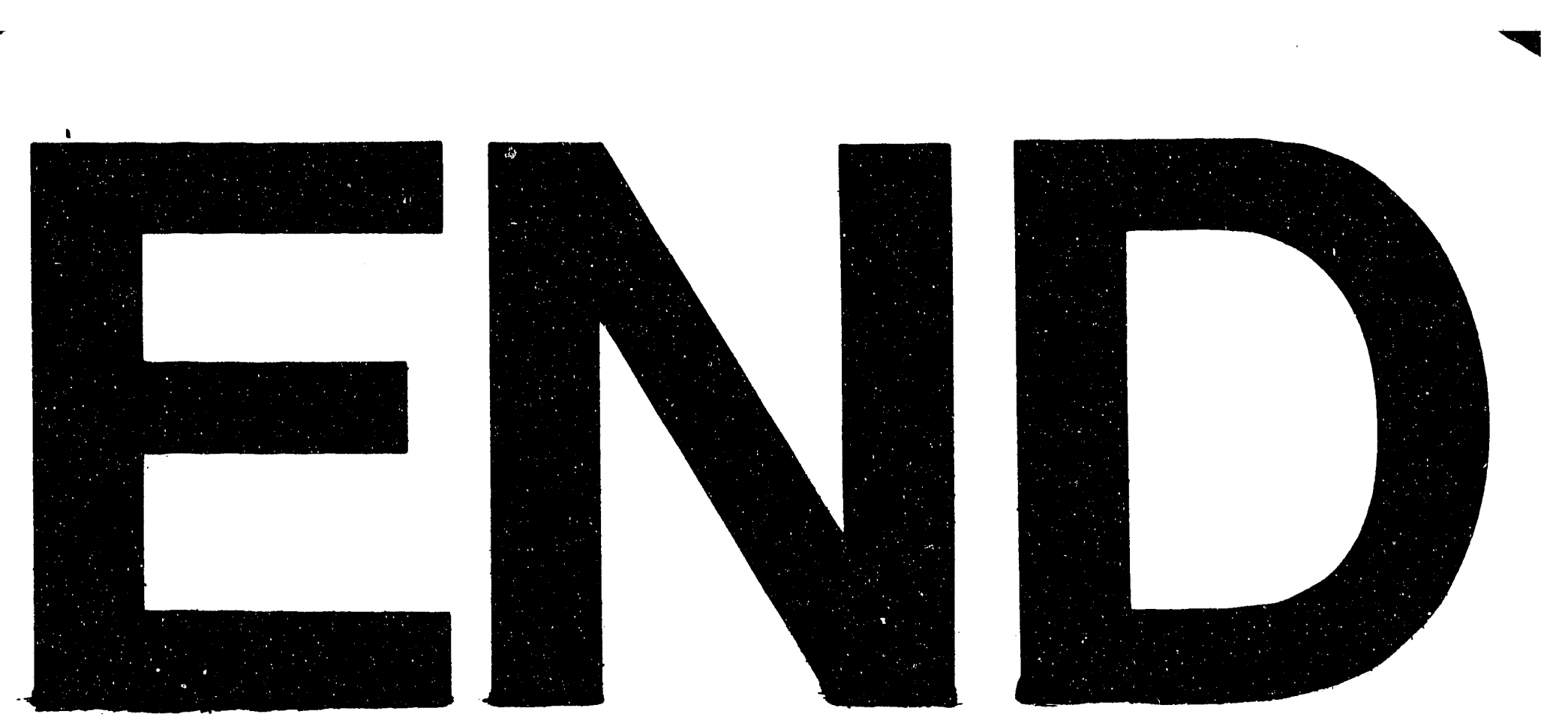

(5)

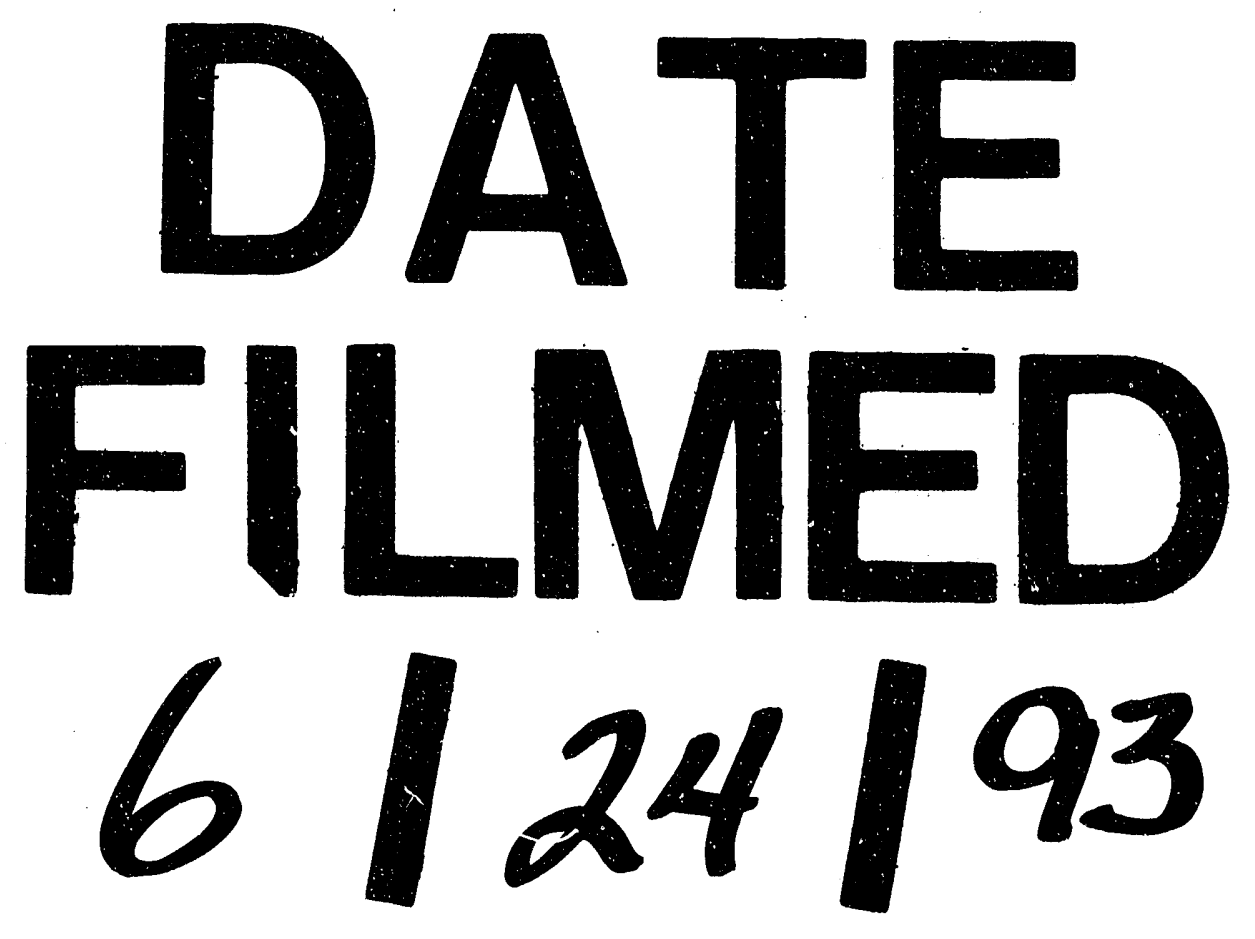


